\title{
Pratiques
}

Linguistique, littérature, didactique

\section{Traitement du figement dans les manuels d'enseignement / apprentissage de FLE pour adultes}

Nathalie Gerber et Olha Luste-Chaâ

\section{(2) OpenEdition \\ Journals}

Édition électronique

URL : http://journals.openedition.org/pratiques/2913

DOI : 10.4000/pratiques.2913

ISSN : 2425-2042

Éditeur

Centre de recherche sur les médiations (CREM)

\section{Édition imprimée}

Date de publication : 15 décembre 2013

Pagination : 228-246

Référence électronique

Nathalie Gerber et Olha Luste-Chaâ, « Traitement du figement dans les manuels d'enseignement / apprentissage de FLE pour adultes », Pratiques [En ligne], 159-160 | 2013, mis en ligne le 30 juin 2016, consulté le 19 avril 2019. URL : http://journals.openedition.org/pratiques/2913 ; DOI : 10.4000/ pratiques. 2913 


\section{Traitement du figement dans les manuels d'enseignement / apprentissage de FLE pour adultes}

\section{Nathalie Gerber, Olha Luste-Chaâ}

CREM, Université de Lorraine

\section{Introduction}

L'apprentissage d'une langue se fait par l'apprentissage des règles de compositionnalité des unités lexicales mais aussi des séquences complètement ou relativement figées qui sont omniprésentes dans la langue et constituent, de ce fait, un domaine qui ne devrait guère être négligé. Dans une réflexion linguistique à visée didactique sur la problématique des phénomènes de figement dans l'enseignement/apprentissage du français langue étrangère (FLE), nous proposons une analyse du traitement du figement dans les manuels de FLE. Cette analyse a un double objectif :

- observer les démarches et activités existantes : discrimination, sensibilisation aux possibilités de variation, activités d'accès au sens et de réemploi, traitement des types de figement en fonction du niveau d'apprentissage ;

— proposer des pistes didactiques pour améliorer leur traitement, dans une réflexion sur 1'approche des préconstruits de la langue à côté de la compositionnalité tout au long de l'apprentissage du français.

\section{Cadre théorique}

\subsection{Critères de figement}

Le phénomène de figement en linguistique du français bénéficie d'une attention soutenue depuis près de trois décennies, dont témoignent de nombreux travaux de J.-C. Anscombre, M. Gross, P. Blumenthal, G. Gross, S. Mejri, I. Mel'čuk, etc. Le figement touche à la fois les domaines de la syntaxe, de la sémantique, du lexique et de la pragmatique. C'est à la croisée de ces domaines qu'ont été définis les principaux critères permettant de caractériser la nature du figement d'une séquence phraséologique ( $c f$. notamment G. Gross 1996) :

- la polylexicalité,

- le figement sémantique (1'opacité sémantique et la non-compositionnalité du sens), 
- la non actualisation des éléments constituants (figement référentiel),

- le blocage des substitutions paradigmatiques,

- le blocage des possibilités transformationnelles (figement syntaxique),

- la portée du figement,

- le degré du figement,

- le défigement.

Les travaux récents (Anscombre et Mejri 2011) montrent cependant que ces traits distinctifs ne se vérifient pas pour toutes les séquences figées (SF) et que le figement est un phénomène scalaire, les séquences pouvant présenter une rigidité plus ou moins grande selon tel ou tel critère.

Selon G. Gross (1996 : 9), la polylexicalité est le critère principal qui permettrait de décrire les SF. Les locutions figées (idem), les phrasèmes complets (Mel'čuk 2011) ou les unités polylexicales (Mejri 2003) se composent de plusieurs unités lexicales qui peuvent être employées de façon autonome hors de la locution en question: par exemple, prendre le taureau par les cornes. Mais, à l'exception de rares contextes, une fois assemblées, ces unités perdent leur valeur dénotative référentielle et ne sont plus actualisées individuellement, renvoyant à un référent unique (figement référentiel). I. Mel'čuk et al. (1995) accordent aux SF le statut d'unité linguistique au même titre que les mots simples.

Dans de nombreuses SF, on ne peut pas interpréter le sens de la séquence par la combinaison de la signification des constituants, autrement dit par le sens compositionnel. Ainsi, dans l'exemple cité supra, le sens propre des lexies prendre, taureau et cornes ne permet pas à un locuteur non natif d'en déduire le sens de l'expression : «S'attaquer de front à une difficulté ». Cette expression a donc un sens non-compositionnel, elle est sémantiquement figée. En outre, les SF n'admettent pas de substitutions paradigmatiques : le fait de remplacer pieds par mains dans Mettre les pieds dans le plat («Intervenir brutalement sur une question délicate », expression figée, sens non-compositionnel), ce qui donne Mettre les mains dans le plat (sens compositionnel, tous les éléments sont actualisés), supprime le figement et la non-compositionnalité sémantique de l'énoncé. La forme Mettre le pied dans un plat ne sera pas non plus perçue comme figée ou renvoyant à la notion de manque de tact d'une personne.

La non-compositionnalité du sens est liée à l'opacité sémantique qui indique que le sens de la séquence n'est pas transparent ni prédictible, comme dans prendre son pied, par exemple. Mais l'opacité de sens ne va pas toujours de pair avec la non-compositionnalité (Mel'čuk 2011 : 51, Mejri 2011 : 76) : dans ne dormir que d'un oil, la transparence de la métaphore permettrait au locuteur de deviner le sens véhiculé, ne serait-ce qu'approximativement. L'opacité sémantique est donc un critère graduable allant du sens complètement opaque (C'est la fin des haricots) au plus ou moins transparent (tenir sa langue).

Une autre caractéristique de nombreuses SF est le figement syntaxique: elles sont très contraintes syntaxiquement et refusent les possibilités transformationnelles des composants (ordre des mots, passivation, clivage, relativisation, séparations linéaires, etc.), à l'exception des locutions verbales et nominales qui acceptent les flexions. Mais, de nouveau, ce critère est relatif : des SF acceptent certaines transformations syntaxiques, et leurs possibilités de transformations déterminent leur degré du figement.

Ces variations syntaxiques posent le problème de la portée du figement. Dans de nombreuses expressions, les limites de la SF sont évidentes, dans d'autres, elles prêtent à discussion. G. Gross (1996 : 38) définit la portée de figement comme «1'éten- 
due, calculée en nombre de mots, de la suite affectée par le figement. L'indication du début et de la fin des séquences contraintes est indispensable [...] ». M. H. Svensson (2004 : 34) élargit cette définition en précisant que dans la portée du figement devraient être inclus le noyau de la séquence et les éléments qui peuvent être modifiés sans porter atteinte au sens de la SF (adjectifs possessifs, pronoms compléments, etc.). L'auteure donne l'exemple des expressions avec le mot gré. Elle présente d'abord au gré de comme expression à structure complètement figée, puis à mon/son/leur grécomme expression acceptant le paradigme des adjectifs possessifs, avec un noyau à_gré et un schéma de portée présentée sous forme de à POSS gré. Enfin, elle analyse de notre plein gré, de son propre gré, de son plein gré et de plein gré comme expressions relevant du même noyau, de _ gré, mais dont la portée du figement s'étend sur toute la structure de \{POSS / plein $\}$ gré ou de POSS propre gré.

Cette conception de la portée du figement rejoint le travail d'E. T. Montoro del Arco (2011) sur les locutions à cases libres et à cases vides. La case libre est indispensable au fonctionnement grammatical de l'unité de sens et obligatoire pour compléter la signification de la SF, elle s'actualise à l'intérieur d'un paradigme restreint et homogène quant à la nature grammaticale des constituants et donne des informations déictiques de temps, d'espace, de personne, etc. (idem: 250 sqq.). Dans les locutions verbales, la case libre est généralement remplie par un pronom corrélé au choix de l'actant (J'ai fait mes adieux / Il a fait ses adieux / *Il a fait des adieux). Dans s'il vous plaît / s'il te plaît, le pronom complément occupe une case libre - il est nécessaire à l'actualisation sémantique de cette séquence - mais dans un paradigme restreint te / vous.

Les cases vides, en revanche, ne sont pas indispensables à la séquence ; les éléments à remplir appartiennent à des catégories lexicales pleines, les possibilités peuvent être nombreuses mais soumises en principe aux contraintes sémantiques (idem:256); 1'insertion de ces éléments n'affecte ni le sens ni le figement de la séquence de base. Certaines séquences sont plus sémantiquement contraignantes que d'autres par rapport au choix de l'élément de remplissage. À titre d'exemple, la locution verbale en avoir marre accepte les flexions, le choix de l'actant est obligatoire sauf pour les emplois infinitifs, J'en ai marre /Ils en ont eu marre / Tu en auras marre / Il y en a marre, mais elle comporte également une case vide qui ne peut être remplie que par un adverbe :Ils en ont eu (vite / vraiment / parfois) marre. Dans ce paradigme, même si la nature sémantique des éléments est variable, leur nature grammaticale est restreinte, ce qui n'est pas attesté dans les dictionnaires et pose problème aux non natifs à l'encodage.

En nous appuyant sur les propos ci-dessus, nous adopterons la notion de portée du figement définie comme l'étendue de la suite affectée par le figement incluant sa partie fixe et non modifiable (le noyau), la/les case(s) libre(s) indispensable(s) à son fonctionnement correct, ainsi que d'éventuelles cases vides variant au gré de l'environnement discursif de la SF.

Le critère de degré du figement est lié à tous les critères décrits supra. A cause du caractère scalaire du figement, des variations ou transformations (im)possibles des $\mathrm{SF}$, le degré de figement est difficile à évaluer et à définir. A des fins d'analyse et de classification, nous distinguerons trois degrés dans le continuum du figement :

- le figement complet caractériserait une séquence qui n'accepte aucune transformation morphosyntaxique, excepté les variations relevant de la syntaxe catégorielle et imposées par les catégories grammaticales, qui est figée au niveau sémantique et référentiel et dont le sens est généralement non-compositionnel;

- le semi-figement concernerait les séquences qui acceptent un certain nombre 
de transformations syntaxiques, dont le sens peut être non-compositionnel ou transparent, et dans lesquelles les éléments sont néanmoins soumis à des contraintes sémantiques ou syntaxiques (le cas des collocations);

- les séquences libres suivent la logique compositionnelle de la langue, chacun de leurs éléments est actualisé et elles acceptent généralement toutes les transformations morphosyntaxiques.

\section{2. État des lieux en didactique du FLE}

Dans les pratiques de classe, la présence de SF non compositionnelles est une difficulté premièrement en compréhension qui peut entraîner une perte de sens parfois secondaire mais souvent significative du texte. Quand les notes de bas de page ne viennent pas à la rescousse de l'apprenant, c'est à l'enseignant d'expliquer que la source de leur incompréhension n'est pas seulement due à des mots qu'ils ne connaissent pas mais à une SF, que l'enseignant se chargera de paraphraser, en passant ou non pas une démonstration par défigement du non-sens du sens compositionnel. En production aussi bien orale qu'écrite, les enseignants consciencieux passent un temps certain à corriger en disant, "C'est bien, mais ce n'est pas comme ça qu'on dit », reconnaissant l'effort de composition d'un énoncé mais aussi la non idiomaticité de la production. Pour acquérir cette idiomaticité, les manuels proposent des modèles de documents écrits ou de conversations; la production à partir de ces modèles est rigide et stéréotypée chez les apprenants qui suivent de près le modèle; pour les apprenants désireux de personnaliser davantage la production, leurs efforts de créativité n'échappent pas au « C'est bien, mais ce n'est pas comme ça qu'on dit ». L'apprentissage des SF se fait alors principalement par erreur-correction, à tâtons, au risque de décourager certains apprenants et de renforcer l'idée qu'apprendre une langue est difficile et qu'il faut être doué pour les langues pour comprendre et se faire comprendre et parvenir à un niveau d'aisance satisfaisant.

Ces difficultés d'enseignement/ apprentissage font l'objet de réflexion dans la littérature spécialisée et plusieurs pistes didactiques intéressantes ont déjà été proposées (Galisson 1983, Vittoz 1989, 2005, Mel' čuk 1993, González Rey 2008, Cavalla 2009 , etc.). Notons en premier l'ouvrage de R. Galisson, Des mots pour communiquer (1983), précurseur dans le domaine de la didactique des SF. En partant de la constatation des difficultés que rencontre l'apprenant dans la maîtrise de ces formes lexicales complexes, l'auteur propose aux enseignants une approche créative, basée sur une démarche onomasiologique, de l'accès au sens des expressions " figuratives » (1983:90) : la création d'un auto-dictionnaire d'encodage, résultat d'un travail individuel avec les mots et le(s) dictionnaire(s) de langue :

«L'enseignant doit se contenter de fournir à l'apprenant des méthodes et des outils d'élucidation du sens, et de découverte de l'usage des mots. Il n'a pas à court-circuiter l'effort de l'apprenant à ce niveau ; le vocabulaire est davantage un problème d'apprentissage, d'investissement personnel, que de l'enseignement. » (idem : 36)

En s'inspirant de la méthode ci-dessus, M. B. Vittoz-Canuto fait paraître en 1989 un ouvrage à visée didactique abordant plusieurs questions : les critères de définition des expressions figées, les problèmes que celles-ci posent aux apprenants dans leur accès à une langue étrangère, la présentation du non-compositionnel dans les dictionnaires et les stratégies d'accès au sens des locutions verbales. Quelques pistes didactiques y sont présentées : le regroupement par champs (par exemple, les expressions contenant les noms de parties du corps, fréquentes en français), le rôle du contexte et de l'isotopie dans la présentation des locutions en décodage, une approche culturelle contrastive (reprise et développée dans Vittoz 2005). 
Citons enfin l'ouvrage d'I. González Rey (2008) et sa méthode Le Français idiomatique, la seule, à notre connaissance, exclusivement dédiée à l'enseignement/ apprentissage des unités phraséologiques (onomatopées, collocations, expressions figées, proverbes, formules routinières, expressions familières, etc.) en FLE. Une multitude d'exercices répartis en deux niveaux (A1-A2) ont pour objectif de familiariser l'apprenant avec ces unités et d'amorcer leur acquisition par le biais des activités centrées sur les composants, la syntaxe et l'aspect sémantique des SF. Une approche culturelle contrastive y est également présente et encourage l'apprenant à traduire et à chercher des équivalents dans sa langue maternelle. Cette méthode ambitieuse et assez complète représente néanmoins un défaut de taille : dans la plupart des exercices, les collocations, les expressions imagées et même les formules routinières apparaissent complètement décontextualisées.

Quant aux collocations, la nécessité de leur réserver une place importante en cours de langue étrangère a souvent été soulignée par les didacticiens. Mais de nombreuses études, qui traitent de la description et des tendances de l'acquisition des collocations ou des pistes didactiques, concernent dans leur grande majorité l'anglais et manquent aux enseignants et apprenants du FLE. Toutefois cette situation est en train de changer grâce à l'intérêt que le phénomène suscite parmi les chercheurs français depuis une dizaine d'années (Tutin et Grossmann 2002, Binon et Verlinde 2003, Grossmann et Tutin 2003, Calaque et David 2004, Calaque 2006, Cavalla 2008, Grossmann 2011 pour l'état des lieux en didactique du lexique). Des outils destinés à faciliter cette tâche pour les enseignants et à aider les apprenants ont également vu le jour. Pratiquement tous ces auteurs insistent sur le fait que l'acquisition/ apprentissage des collocations ne peut pas être uniquement involontaire à partir de l'input; par ailleurs, nombreux sont ceux qui prônent la mémorisation des collocations comme des blocs lexicaux à la manière des locutions figées.

\subsection{Catégories de SF pour la didactique du FLE}

Nous avons arrêté des catégories pour l'analyse des SF en fonction de la récurrence de certaines SF dans les manuels et de leur pertinence en didactique du FLE. Si ces catégories ne sont pas étrangères à celle de la lexicologie, et notamment à la «typologie universelle des phrasèmes » de Mel'čuk (2011), elles diffèrent quelque peu. A la différence de la lexicologie, les catégories telles que nous les présentons n'ont pas pour but de classer systématiquement les SF et de trancher pour les SF qui sont à la frontière de plusieurs catégories. Il s'agit plutôt de saisir des types de SF caractérisées par des traits définitoires qui appellent des stratégies d'enseignement/ apprentissage différentes. Les catégories que nous retenons pour l'analyse sont les pragmatèmes, les locutions adverbiales, les collocations, les proverbes et les expressions imagées.

Les pragmatèmes sont des SF contraints par rapport à une situation d'énonciation (terme que nous préférons à situation extralinguistique utilisé par Mel'čuk (2011)). Ne quittez pas! n'a le sens de «Ne raccrochez pas le combiné téléphonique ! » que dans une conversation téléphonique. Dans d'autres contextes, Ne quittez pas ! serait erroné dans la mesure où quitter est un verbe transitif. Les pragmatèmes sont nombreux dans la langue orale, ils apparaissent surtout dans les interactions normées, les formules de politesse, les routines conversationnelles (Klein et Lamiroy 2011). On les trouve en grand nombre également à l'écrit dans des genres contraints comme la correspondance, les écrits professionnels : Par la présente, ordre du jour. Les pragmatèmes nécessitent des traitements de décodage et d'encodage en situation de com- 
munication dès le niveau A1 pour pouvoir interagir avec efficacité. Ça va ? est un de ces pragmatèmes communément appris dans les premiers échanges en FLE.

Nous entendons par locutions adverbiales, d'une part, les SF de causalité (à cause de), d'opposition (en revanche), de concession (tout de même), de but (de sorte que), de condition (à condition que), d'autre part, les SF qui sont des marqueurs de 1'organisation textuelle (en effet, par exemple) et des marqueurs d'énonciation (à mon avis, sans doute), et enfin les SF spatio-temporelles et les quantifieurs. Ces distinctions sémantico-pragmatiques suivent des catégories fonctionnelles qu'on retrouve dans l'organisation des contenus des manuels : expression de la cause, expression de la condition, expression du temps, expression du lieu, donner son avis, écrire un texte argumentatif. Les connecteurs et les marqueurs d'énonciation sont caractérisés par leur haut degré de figement. Si l'on trouve des locutions figées parmi les adverbiaux spatio-temporels et les quantifieurs (de temps en temps, à ce moment-là, une foule de), on $\mathrm{y}$ trouve principalement ce qui relève de matrices lexicales au sens d'Anscombre (2011) : à demain / à l'année prochaine / à tout à l'heure, à côté de/ à droite de/ en face de/ au fond de, un kilo de/une bouteille de/ un peu de. A ces adverbiaux, on peut ajouter les locutions prépositionnelles de manière qui fonctionnent également selon des matrices lexicales : à la française / à l'anglaise / à la va-vite, par obligation / par nécessité /par devoir, avec amour / avec joie / avec plaisir, sans y penser / sans y prendre garde.

Depuis l'intégration de documents authentiques et le développement du FOS, les collocations sont apparues massivement dans les manuels. Sous l'étiquette collocation, nous classons, en reprenant en partie les traits définitoires de Tutin et Grossmann (2002), toute SF binaire, constituée d'une base à fonctionnement compositionnel et d'un collocatif (une lexie ou un syntagme) sélectionné en fonction de la base pour exprimer un sens particulier. Le sens du collocatif peut-être opaque, comme dans une peur bleue, transparent, comme dans annoncer une nouvelle, métaphorique et transparent, comme dans construire une argumentation; le collocatif peut aussi subir un affaiblissement sémantique comme dans les constructions à verbe support : faire le ménage, faire des études, avoir peur, avoir froid. Le choix du collocatif se fait dans un paradigme fermé ou restreint pour exprimer un sens particulier : pour parler d'une année de 366 jours, il n'y a que le collocatif bissextile qui convienne ; saisir des données peut être remplacé par rentrer des données. Dans l'analyse des collocations, nous nous intéressons également au réseau de collocatifs admis par une base ; ces collocatifs peuvent caractériser la base avec des sens différents dans un paradigme restreint : le réseau des collocatifs de café (la boisson) est noir, au lait, serré, léger, rallongé, filtre, expresso, italien, turc.

Nous analyserons le traitement fait aux expressions imagées et aux proverbes qui ont en commun, en plus de leur caractère figé, de relever de procédés stylistiques et d'être considérés comme des reflets d'une culture (Vaguer 2011). Les expressions imagées sont des SF qui prennent la forme d'un syntagme nominal ou d'un syntagme verbal, et dont la signification repose sur un procédé métaphorique ou comparatif: être bête comme ses pieds, avoir un mot sur le bout de la langue, un chèque en bois. Les proverbes, ou clichés dans la terminologie de Mel'čuk (2011), sont des énoncés complets, syntagmatiquement figés mais sémantiquement compositionnels : C'est en forgeant qu'on devient forgeron. Ce sens compositionnel est couplé à un sens formulaire (Tamba 2011), il en est l'emblème ; la vérité générique de la situation proverbiale est transférée métaphoriquement à la situation d'énonciation. Les expressions imagées et les proverbes posent plusieurs problèmes didactiques : le décodage et l'encodage, l'emploi dans des situations appropriées, l'appréhension de la dimension culturelle. 
Ces catégories ne sont pas hermétiques : on trouve des collocations qui sont des expressions imagées, un argument de poids, des pragmatèmes qui contiennent des collocations, J'apprécie beaucoup!, ou sont des locutions adverbiales, Avec plaisir!; les quantifieurs peuvent être analysés aussi bien sous l'angle d'une matrice lexicale, de la collocation ou même, pour certains, de l'expression imagée : une avalanche de questions. Des SF cumulent ainsi les caractéristiques de plusieurs catégories.

Dans chacune de ces catégories, les SF n'ont pas le même degré de figement : la question du décodage dans le cas des SF non-compositionnelles et opaques et la question de la portée des SF, essentielle dans le décodage et l'encodage, leur sont transversales.

\section{Analyse des manuels}

\subsection{Le corpus}

Le corpus ( $c f$. Annexe 1) est constitué de 42 manuels d'apprentissage du FLE, édités par CLE International, Didier, Hachette Français langue étrangère et les Presses universitaires de Grenoble (PUG), maisons d'édition spécialisées dans le FLE. Les manuels du corpus ciblent tous le public « grands adolescents et adultes ». Le corpus couvre tous les niveaux d'apprentissage, de A1 à C2 du Cadre européen commun de référence pour les langues $(C E C R L)$. Si les niveaux $\mathrm{A} 1, \mathrm{~A} 2, \mathrm{~B} 1$ et $\mathrm{B} 2$ sont à peu près équitablement représentés, les niveaux $\mathrm{C} 1$ et $\mathrm{C} 2$ ne sont représentés que par deux manuels, Le chemin des mots [29] ${ }^{(1)}$ et Le français pour les sciences [25]. Ce déséquilibre s'explique par le faible nombre de manuels élaborés pour ces niveaux.

Nous avons choisi d'analyser le figement dans différents types de manuels : des méthodes générales et leurs cahiers d'exercices, des manuels de vocabulaire, des manuels de grammaire et des manuels d'apprentissage du français sur objectifs spécifiques (FOS). Ce choix permet d'explorer le figement dans différentes approches de la langue : approches grammaticale et lexicale, dans la perspective de l'acquisition d'une langue générale « du quotidien », ou d'une langue de spécialité. Pour chaque type de manuel, nous avons sélectionné un ou deux titres de chaque maison d'édition avec l'ensemble des niveaux déployés sous ce titre, à l'exception de certains titres pour des raisons de taille du corpus. À noter que Didier ne propose pas de manuel de vocabulaire, et que là encore pour des raisons de limitation du corpus, nous n'avons pas analysé de manuel de vocabulaire de chez Hachette.

Les manuels ont été choisis également en fonction de leur actualité : ils ont été réalisés pour la plupart dans la décennie passée. Seuls Grammaires progressives [4042] et Vocabulaire progressif [33-35] remontent aux années 1990 ; ils restent toutefois d'actualité dans la mesure où ils ont été depuis réédités ou réimprimés et n'ont pas été éclipsés par d'autres manuels du même type chez CLE International. Sur la période couverte par le corpus, il y a eu trois évolutions didactiques notables : l'intégration des principes du CECRL, l'approche actionnelle et le développement du FOS. Ces trois évolutions s'inscrivent dans la continuité de l'approche communicative.

(1) Les manuels sont cités dans le texte sous forme abrégée et sont accompagnés d'un numéro entre crochets qui correspond à leur numéro dans les tableaux en annexes. Pour leurs références complètes, se référer à l'Annexe 1 . 


\subsection{Résultats}

\subsubsection{Terminologie et discrimination}

Nous nous sommes intéressées à la terminologie utilisée dans les manuels de FLE pour désigner les phénomènes de figement et aux procédés graphiques employés pour discriminer les SF.

L'abondance terminologique dans la littérature scientifique contraste avec celle des manuels de FLE. On trouve dans la plupart des méthodes expressions, expressions imagées et proverbes. Apparaissent également les termes suivants : expressions à connaître (Travailler en français [27-28]), Comment le dire? (Communication progressive [7-8]), on dit (Grammaire du français [36]), expressions de localisation (Grammaire des premiers temps 1 [38]) expressions particulières au style oral (Français par les textes 1 [31]), formules d'appel et de politesse, formulations principales pour la correspondance écrite (Echo B2 [15]). Cette terminologie est en partie révélatrice du traitement du figement dans les manuels de FLE. Elle est le reflet du peu de distinctions faites entre pragmatèmes, collocations, locutions figées ; elle montre par ailleurs le traitement spécifique réservé aux expressions imagées et proverbes, à certaines formules de l'oral et à la correspondance.

Le chemin des mots, qui ne s'inscrit pas directement dans l'approche communicative mais qui veut la compléter par un apport de connaissances linguistiques sur la construction du lexique et des expressions du français, emploie une terminologie plus détaillée : expressions latines, collocations, comparaison, métaphores lexicalisées, etc.

Dans les manuels, les SF sont souvent discriminées par une typographie en gras ou en italique, apparaissent parfois entre guillemets, sont signalées par un renvoi en note de bas de page, sont mises en valeur dans des listes, des tableaux, des encadrés ou des étiquettes. La mise en évidence des SF permet de sortir d'une vision monolexicale de la langue, même si elle reste prédominante. Par contre cette discrimination est lacunaire et arbitraire : certaines SF sont discriminées, d'autres non, la portée discriminée des SF n'est pas toujours juste, le degré de figement et les variations possibles ne sont pas indiqués. Cependant la qualité du traitement des SF varie selon les catégories.

\subsubsection{Locutions adverbiales}

Dans tous les manuels où elles apparaissent, les locutions adverbiales de causalité, de condition, d'opposition et de temps et les locutions propositionnelles de lieu sont discriminées avec justesse et sont l'objet, selon les niveaux, d'exercices de compréhension, d'analyse de leur fonctionnement et d'activités de réemploi. Ces locutions sont traitées en général dans les rubriques grammaticales des méthodes générales ou FOS et dans les manuels de grammaire. Certaines rubriques ou leçons de grammaire abordent les moules syntaxiques de ces locutions adverbiales ; La grammaire des premiers temps 2[39] accorde par exemple une leçon p. 59 aux locutions prépositionnelles dans un chapitre sur la caractérisation.

\subsubsection{Pragmatèmes}

Des pragmatèmes, de degré de figement variable, apparaissent et sont discriminés massivement dans les manuels qui abordent l'enseignement du FLE par actes de parole dans des situations de communication. Communication progressive, Scénario [18-19], Objectif express [20-22], Travailler en français, Le français par les textes, Vocabulaire progressif contiennent des exemples d'interactions orales et de correspondances et autres documents écrits d'où sont extraits, dans des encadrés ou des ru- 
briques connexes, des formulations correspondant à des actes de parole. Ces formulations sont plus ou moins contraintes par les rites d'interaction (formules de politesse), par des schémas discursifs normés par des habitus (schémas conversationnels chez les commerçants, dans les services administratifs, dans les écrits professionnels). Les énoncés scientifiques sont également normés et font l'objet d'activités de décodage et d'encodage dans le Français pour les sciences. Dans A propos [1-3-5], Echo [9-11-13-15], ou Le Nouvel Edito [17], les pragmatèmes sont présents mais de manière moins massive que dans les manuels cités précédemment; ces manuels sont centrés davantage sur le développement de compétences de compréhension et expression de textes explicatifs, narratifs, descriptifs et argumentatifs où les pragmatèmes se font plus discrets. Un signe de la prise en compte des pragmatèmes dans l'enseignement du FLE est l'apparition dans certaines méthodes de modèles d'écrits (Travailler en français) et de mémento d'actes de parole (Objectif Express, A propos A1, $A 2$ [1-3]) en fin d'ouvrage.

Le chemin des mots, axé sur une approche linguistique, Le français du monde du travail [24], élaboré sur un mode informatif, et Dites-moi [30], basé sur des dossiers thématiques culturels en vue de développer l'expression orale, ne discriminent aucun pragmatème.

Dans Grammaires pratique [37], Grammaire progressive et Grammaire du français, des phrases qu'on peut apparenter à des actes de parole servent à illustrer et à manipuler des éléments grammaticaux; mais la brièveté des dialogues et l'absence de contexte pour des phrases isolées ne permettent pas de les envisager comme pragmatèmes. Cependant, les pages « Variations - Réemploi » de Grammaire du français contiennent quelques documents proches de l'authentique, contenant des pragmatèmes : les faire-part avec leurs formules « ont le plaisir de vous annoncer le mariage de », « sont heureux de vous annoncer la naissance de » (p. 28), par exemple, qui sont l'objet d'activités d'imitation. Grammaire progressive 3 [42] présente de petits corpus de pragmatèmes, Défense de fumer, Coiffeur pour dames (p. 16), pour illustrer des cas d'absence d'article devant le nom. De manière générale, certains chapitres (sur l'infinitif, le conditionnel, le subjonctif et la nominalisation) favorisent l'apparition de pragmatèmes (formules de recette de cuisine, expression du conseil et de la recommandation, tournures impersonnelles ou personnelles de point de vue, titres de presse et panneaux de signalisation).

Un problème récurrent posé par les pragmatèmes est la présentation de leur degré de figement et de leurs variations possibles. Ces indications apparaissent plus souvent au niveau débutant et se font plus rares au niveau intermédiaire et disparaissent au niveau avancé. Dans les encadrés d'actes de parole, les mémentos ou les modèles de lettres, des signes graphiques indiquent des cases libres et des cases vides :

Répondre suite à la présentation d'une personne (A propos A1 [1], Mémento, p. 106) :

1. (Je suis) enchanté(e) de faire votre connaissance.

2. (Je suis) ravi(e) de vous rencontrer.

Décrire des symptômes chez le médecin ou à la pharmacie (Objectif express 1 [20], p. 169):

\section{J'ai (très) mal àlaux (+ partie(s) du corps).}

Se plaindre (Objectif express 2 [22], p. 175)

4. Ce n'est quand même pas normal. 
5. C'est la deuxième fois que ça arrive.

6. Comment ça!

7. Vous plaisantez!

Mais ces indications manquent de rigueur et paraissent parfois aléatoires. Pour plus de justesse et de distinction entre case libre (que nous proposons de mettre entre crochets) et case vide (que nous laissons entre parenthèses), on devrait trouver :

1(a) (Je suis) enchanté[e] (de faire votre connaissance).

2(a) Je suis) ravi[e] $\{$ de [vous/te] rencontrer

$\{$ de faire [votre/ta] connaissance

3(a) J'ai (très) mal àlaux [+ partie(s) du corps].

4(a) Ce n'est (quand même/vraiment) pas normal.

5(a) C'est la [deuxième/troisième/quatrième...] fois que ça arrive.

6(a) Comment ça! ${ }^{\circ}$

7(a) Vous plaisantez! / Tu plaisantes!

On pourrait indiquer par un rond en exposant (ex. 6( $a)$ ) les quelques pragmatèmes invariables. Si les apprenants des niveaux intermédiaires et avancés peuvent deviner des variations possibles en fonction de leur connaissance de la langue, le degré de figement ne peut qu'hasardeusement être déduit. On peut en effet dire Enchanté ! mais on ne peut pas dire simplement *Ravi! lors de présentations. Vous plaisantez! existe à la deuxième personne du singulier, mais Tu parles! n'existe qu'à la deuxième personne du singulier. Sans être exhaustif dans la présentation de variations, l'indication du figement total, de cases libres et de cases vides, et ce même au niveau avancé, semble être incontournable, au moins sous forme de sensibilisation, pour la bonne acquisition des pragmatèmes et des SF en général.

\subsubsection{Collocations}

Des collocations sont visibles dans certains documents supports tels que les tableaux, les graphiques, les affiches, ou autres documents où la mise en page laisse voir des unités sémantiques polylexicales : le bon de commande dans Objectif express 2 (p. 54) laisse appréhender comme unités frais de port, net à payer, total TVA, et dans les sondages « Êtes-vous accro aux nouvelles technologies? » dans Echo A1 [9] (p. 94), on trouve détachées, entre autres, les collocations créer des documents, aller sur Internet, jouer à des jeux vidéo, jouer en réseau. Elles sont visibles également dans des listes de vocabulaire ou d'expressions où elles sont minoritaires. On ne trouve pas encore d'index ou de mémento de collocations. La Grammaire des premiers temps fait apparaître entre parenthèses des constructions à verbes supports et autres collocations verbales à conjuguer. Des textes, la plupart du temps explicatifs et informatifs, présentent, en gras, mots et expressions clés à retenir par rapport au thème abordé. Parmi ces unités discriminées, les collocations sont nombreuses. On trouve ce procédé dans les manuels de FOS, Le français pour les sciences, Le français du monde du travail, dans les pages « Repères culturels » d'Objectif express, ainsi que dans les leçons de Vocabulaire progressif. Dans les textes supports, on trouve sporadiquement ou en grand nombre des collocations qui ne sont pas discriminées. A propos et Le nouvel édito n'usent pas de procédé de discrimination des collocations dans les textes. On en trouve toutefois mises en valeur dans des activités de compréhension et production: dans 1'activité 11 p. 76 de A propos B1-B2, Cahier $d$ 'exercices [6], on demande de souligner les thèmes abordés dans l'interview parmi une liste de syntagmes nominaux où l'on trouve outils de travail, durée hebdomadaire de travail, conditions de travail, avantages en nature, perspectives de carrière, qualités exigées, etc. 
Quand les collocations apparaissent, elles sont souvent regroupées par champs lexicaux et parfois par champs sémantiques. L'activité 12 p. 123 de $A$ propos B1-B2, Cahier d'exercices propose une réflexion sur la transparence ou l'opacité d'expressions de forme Nom + de famille, comme Affaires de famille, Air de famille. Elles sont aussi déployées sous forme de paradigmes, comme dans la leçon «Les différents types de sociétés en France» dans Le Français du monde du travail (p. 8): sociétés de capitaux, sociétés de personnes, sociétés à responsabilité limitée, etc. Ces déploiements de paradigmes sont autant des apports lexicaux qu'un apport de connaissances sur le domaine.

Si ces collocations sont discriminées, expliquées, 1'objet d'observation et de réemploi, elles sont peu l'objet d'activités de reconstitution (par appariement ou sélection) ( $c f$. Annexe 2). A la suite de l'activité 11 décrite ci-dessus, il est demandé de prendre des notes, de reconstituer l'interview à deux et de rédiger une dizaine de questions pour interroger quelqu'un sur son travail (A propos B1-B2 [5], p. 73); ces activités nécessitent le réemploi des collocations dans différentes situations : prise de notes, restitution d'informations, rédaction d'un questionnaire. Mais aucune activité ne porte sur la reconstitution des collocations.

Les grammaires proposent des approches directes ou indirectes de certains types de collocations. Les leçons sur la place de l'adjectif, la nominalisation, l'absence d'article et les verbes supports font apparaître dans la Grammaire des premiers temps, Grammaire progressive 2 et 3 des collocations.

Le chemin des mots consacre un chapitre à la collocation mais on la retrouve également dans les chapitres suivants : "Les mots construits par composition populaire», « Synonymie et précision », « Comment les mots changent de sens », « Comparaison », «Les métaphores lexicalisées ». Ces chapitres permettent d'étayer 1'acquisition des collocations par une connaissance sur les collocations.

Outre le manque d'activités de reconstitution, on peut constater le caractère aléatoire de la discrimination des collocations qui s'accompagne de problèmes de portée et de non indication du degré de leur figement. Dans les fiches de vocabulaire de Echo B2, Cahier personnel [16] (p. 6 et 8), sont intégrés parmi les unités monolexicales et des unités polylexicales :

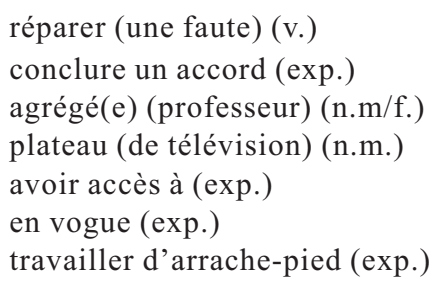

On peut se demander ce qu'indiquent les parenthèses et la désignation (exp.). Aucun autre manuel ne propose de discrimination plus juste ou plus avancée. Vocabulaire progressifqui discrimine plus ou moins systématiquement et avec justesse les collocations de ses leçons en gras, ne les distingue pas des autres unités sémantiques à apprendre, également discriminées en gras, et n'indique pas toujours les variations possibles.

\subsubsection{Expressions imagées et proverbes}

On trouve dans les différents types de manuels des expressions imagées, disséminées sporadiquement dans les documents, dans les rubriques de vocabulaires, dans des exemples grammaticaux ou des rubriques culturelles : Bon vent, les éoliennes (titre d'un document, Echo B1.1 [13], p. 78), le coup de foudre (leçon « Les relations, les 
sentiments » de Vocabulaire progressif 1), Il est doux comme un agneau (exemple dans le chapitre "Comparaison» de Grammaire progressive 3), La barbe («Repères culturels : Joindre le geste à la parole », Objectif express 2, p. 119). Ces expressions sont parfois discriminées, parfois expliquées, mais souvent non traitées ( $c f$. Annexe 3). Le traitement ou le non traitement dans un même manuel de ces expressions semble aléatoire.

A côté de ces apparitions et traitements irréguliers, des expressions imagées sont présentées dans des rubriques lexicales spécifiques ou sont traitées dans des leçons à part: "Quelques expressions imagées » dans Vocabulaire progressif 2 et 3, "Quelques expressions » dans Dites-moi, «Expressions » dans les pages de vocabulaire et « Expressions imagées » (p. 174-175) dans Le nouvel Edito. La présentation de ces expressions est accompagnée parfois d'une paraphrase et d'un exemple de mise en contexte (Vocabulaire progressif 3, p. 124), d'exercices d'association à une explication ou à une situation (Le nouvel Edito, p. 174-175), ou à une demande de commentaire (Dites-moi).

Dans les livres de grammaire, on trouve des petits corpus d'expressions imagées pour illustrer ou manipuler la structure comparative avec comme ou l'emploi des prépositions de lieu : «Comme : créativité » (p. 197) et «Prépositions : échanges/créativité » (p. 236) dans Grammaire des premiers temps 1, la rubrique Parlons ! du chapitre "Comparaison» de Grammaire pratique; dans la Grammaire des premiers temps 1 , le traitement des expressions imagées comparatives s'accompagne en plus d'une approche culturelle comparative avec la langue maternelle de l'apprenant.

Les expressions imagées sont également traitées dans des rubriques culturelles autour d'un thème ou d'un mot récurrent dans les expressions imagées françaises : expressions imagées autour de la pomme de terre et du pain dans Scénario 2 [19] rubrique « Culture, cultures » p. 62-63; Le nouvel édito et Dites-moi font de cette approche culturelle thématique une approche privilégiée et quasi-systématique. Les expressions imagées sont parfois reléguées au cahier d'exercices comme dans $O b$ jectif express 1 (Unité 10) ou A propos A2 [3-4] (Dossier 6).

Dans la grande majorité des cas, quel que soit le degré de traitement de ces expressions imagées, elles sont souvent accompagnées d'une ou de plusieurs images illustrant l'expression d'une manière littérale; ces images humoristiques, qui sont ou non l'objet d'activités, relèvent d'un procédé de défigement et peuvent permettre une réflexion sur le sens littéral et le sens figuré. Seul A propos A2 (p. 77) aborde les expressions imagées en rapport avec un genre textuel particulier, les titres de faits divers, où elles sont l'occasion de jeux de mots : « Deux serruriers sous les verrous », « Le notaire passe à l'acte ").

Les proverbes sont bien moins présents dans les manuels de FLE ( $c f$. Annexe 3). En plus des quelques proverbes qui apparaissent ici et là dans des documents supports, on en trouve deux illustrant la structure « avant de + infinitif » dans Grammaire des premiers temps 2 p. 181, une demi-douzaine dans Grammaire progressive 3 pour illustrer l'absence d'article (p. 20), quelques-uns dans Scénario 1 [18] p. 88 qui sont l'occasion de défigement par la caricature, d'autres dans la rubrique « Citations et proverbes » de Dites-moi (qui privilégie largement en nombre les citations) accompagnés de la consigne «Quelle est la citation que vous préférez? ». A propos B 1-B2 (p. 126) propose une série de proverbes français et une série de proverbes étrangers autour du thème de l'argent, ainsi qu'une compréhension orale où un homme commente certains de ces proverbes ; le cahier d'exercices associé propose une activité d'association entre les proverbes et leur signification, une activité d'imitation du commentaire de proverbes et une compréhension orale où sont expliquées les différences entre proverbes, dictons et expressions proverbiales. 
Au vu de ces analyses, on constate que les expressions imagées et les proverbes, très sporadiques au niveau débutant, deviennent récurrents ou l'objet de leçons et d'activités aux niveaux intermédiaire et avancé, notamment dans les méthodes et les manuels de vocabulaire, sans être totalement absents des manuels de grammaire et de FOS. Les approches proposées dans les manuels sont variées et complémentaires. Mais on constate aussi le traitement aléatoire et partiel des expressions imagées et des proverbes dans les manuels pris individuellement. Les principales lacunes sont, d'une part, le peu d'activités de reconstitution de ces SF et, d'autre part, le peu d'analyse et de réemploi de ces SF en contexte qui permettrait d'associer ces SF à des registres de langue et à des genres discursifs, d'appréhender leur valeur argumentative et d'apprendre à les insérer dans un discours. Ces lacunes montrent toutefois que la priorité est faite à une compétence passive de décodage de ces SF et non à une compétence active d'encodage. En cela, les manuels restent dans les recommandations du CECRL.

\section{Propositions didactiques}

L'un des objectifs que nous nous sommes fixés est de réfléchir à comment mieux intégrer le phénomène de figement dans l'enseignement / apprentissage du FLE. À la lumière des résultats de l'analyse des manuels, nous voudrions proposer quelques pistes didactiques.

\subsection{Développement d'une compétence active}

Nous avons remarqué que dans les manuels, les activités proposées visant les expressions imagées, les proverbes et les collocations sont généralement basées sur une approche «passive » : l'accès au sens et la mémorisation d'une SF se font à l'aide d'une paraphrase et hors contexte, et sont nettement plus favorisés par rapport aux activités de réemploi ou de production. Notre première proposition porte donc sur le développement d'une compétence active de la maîtrise des SF : pour pouvoir mobiliser celles-ci correctement et sans effort dans un discours spontané oral ou écrit, l'apprenant doit non seulement pouvoir trouver dans son lexique mental la SF correspondant au sens voulu, mais aussi connaître le/les contexte(s) possible(s) de son emploi. Ces connaissances pourraient s'acquérir à partir d'une approche ciblée sur les SF et des observations plus systématiques des documents supports audiovisuels et écrits de différents genres proposés dans les méthodes, avec un repérage guidé des contextes d'emploi. On accordera ainsi une attention particulière aux champs de langue (français du travail, français juridique, français du quotidien, etc.) et aux genres (lettre administrative, article éditorial, conversation amicale, etc.) qui favorisent certains types de SF.

Les documents authentiques représentent une «mine d'or » pour relever et travailler les collocations et d'autres SF en contexte, et ceci à tous les niveaux de maîtrise de la langue. Mais ces documents qui ont fait leur entrée dans les manuels ces dernières années sont sous exploités à cet égard. Voici une liste non exhaustive des activités qui permettraient à l'apprenant de mobiliser plus facilement ses acquis dans les interactions en dehors des cours : 1'illustration systématique d'emploi des SF dans de petites conversations autour des situations quotidiennes en complément des paraphrases pour expliquer le sens de l'expression, une discrimination uniforme des SF dans les supports écrits tout au long de la méthode, un entraînement systématique au réemploi en contextes appropriés et variés, des activités de correction, de reconstructions et d'association (à une situation) de différents types de SF. 


\section{2. Élaboration de fiches lexicographiques à visée didactique}

L'idée de faire élaborer aux apprenants leur propre outil d'apprentissage du lexique et surtout de la phraséologie du français a déjà été soulevée par R. Galisson (1983) et M.-B. Vittoz (1989, 2005, avec des exemples) comme un gage d'un apprentissage réussi. L'auto-dictionnaire a pour objectif de consolider chez l'apprenant les compétences de décodage et de faciliter l'encodage des SF. Nous nous en inspirons pour notre proposition d'introduire dans les méthodes de FLE des «Fiches d'expressions et de formules » (une suggestion de titre) qui seraient présentes dès le niveau débutant (A1-A2).

Au début de l'apprentissage, une telle fiche aurait pour fonction de sensibiliser l'apprenant aux différentes SF en français. Elle comporterait la SF (proverbe, expression imagée, collocation, etc.) relevée avec une indication de contexte, une paraphrase et sa portée complète avec cases libres cases vides et figement total ( $c f$. supra), deux ou trois illustrations de variations. Quelques fiches seraient incluses régulièrement dans le corps du manuel et éventuellement du cahier d'exercices. L'éditeur pourrait parallèlement offrir la possibilité de consulter un plus grand nombre de fiches sur Internet ou sur un CD-ROM.

Aux niveaux intermédiaire et avancé, ces fiches d'exploration du lexique se présenteraient sous une forme plus élaborée avec des indications plus complètes sur les possibles contextes d'emploi, le type de figement sémantique (sens propre, sens figuré, sens opaque), l'attribution des catégories (pragmatèmes, collocations, proverbes, expressions imagées). Dans les annexes des méthodes, au même titre qu'y figurent des précis de grammaire et de conjugaison ou un index lexical, 1'apprenant trouverait des fiches vierges à compléter en autonomie ou pendant une séance consacrée à la phraséologie.

\section{Conclusion}

Au terme de cette analyse du traitement du figement, nous constatons dans les manuels de FLE, d'une part, la présence régulière, à plus ou moins grande fréquence, de pragmatèmes, locutions adverbiales, collocations, expressions imagées et proverbes dans les manuels, des variations de leur fréquence en fonction du type de manuel (méthode, vocabulaire, grammaire, FOS), mais, d'autre part, une terminologie «fourre-tout», leur discrimination aléatoire et approximative, le manque d'analyse de leur emploi en contexte et d'activités de reconstitution, la non précision de leur portée, la non prise en compte des variations possibles. Ces lacunes peuvent notamment avoir des répercussions sur la capacité d'un apprenant à intégrer les SF dans son lexique actif. Enfin, le traitement privilégié accordé aux expressions imagées et proverbes éclipse d'autres séquences partiellement figées comme les collocations, laissant à la charge et au bon vouloir de l'enseignant d'y attirer l'attention de ses apprenants.

Pour pallier ces lacunes, nous proposons quelques pistes didactiques : un code graphique pour indiquer la portée et le degré de figement, des activités de développement d'une compétence active, par l'emploi en contexte notamment, et la réalisation de fiches lexicographiques didactisées. Le développement de ces propositions didactiques nécessiterait des expérimentations avec les différents publics d'apprenants de FLE (selon leurs niveaux, leurs objectifs) sur une longue période, car il s'agit d'intégrer, au-delà des supports, une nouvelle représentation de la langue constituée d'unités monolexicales mais aussi d'unités polylexicales, sujettes à des varia- 
tions. Si le code graphique proposé est élémentaire, sa juste application nécessitera de s'appuyer sur les recherches concernant la portée et la variation des SF ; la constitution d'un recueil de SF par catégories avec application du code graphique pourrait être un projet intermédiaire qui servirait de ressource aux concepteurs de manuels. Les travaux actuels en lexicographie sur des fiches et des dictionnaires de SF pourront servir de ressources et de modèles à didactiser. Le développement d'activités autour des contextes d'emploi des SF devrait s'appuyer sur des analyses interactionnelles et discursives qui ne sont à ce jour que partiellement réalisées.

\section{Bibliographie}

ANSCOMBRE, J.-Cl. (2011), «Figement, idiomaticité et matrices lexicales », in J.-Cl. Anscombre \& S. Mejri (éds), Le figement linguistique : la parole entravée, Paris, Honoré Champion Éditeur, p. 17-40.

ANSCOMBRE, J.-Cl., MEJRI, S. (éds) (2011), Le figement linguistique : la parole entravée, Paris, Honoré Champion, Coll. LEXICA Mots et Dictionnaires.

BINON, J., VERLINDE, S. (2003), «Les collocations : clef de voûte de l'enseignement et de l'apprentissage du vocabulaire d'une langue étrangère ou seconde », La lettre de l'AIRDF, 33, p. 31-36.

CAlaque, É. (2006), «Collocations et image de l'organisation lexicale », Actes du Colloque AFLS(Association for French Language Studies), Didactique / linguistique de corpus, 2-4 septembre 2005, Université de Savoie, Chambéry.

Calaque, É., David, J. (2004), Didactique du lexique : Contextes, démarches, supports, Bruxelles, De Boeck.

CAVAlla, C. (2008), «Les collocations dans les écrits universitaires : un lexique spécifique pour les apprenants étrangers », in $\mathrm{O}$. Bertrand et I. Schaffner (éds), Apprendre une langue de spécialité : enjeux culturels et linguistiques, Paris, Éditions École Polytechnique, p. 93-104.

- (2009), «La phraséologie en classe de FLE », Les Langues Modernes 1, vol. 103, [en ligne]. Disponible sur le site Web de la revue :www.aplv-languesmodernes.org (Page consultée le 25 mars 2013).

CONSEIL DE L'EUROPE (2001), Cadre européen commun de référence pour les langues : apprendre, enseigner, évaluer. Paris, Didier.

GALISSON, R. (1983), Des mots pour communiquer, éléments de lexicométhodologie, Paris, CLE International.

GONZÁLEZ REY, I. (2008), La didactique du français idiomatique, Fernelmont : E.M.E. Éditions.

GROSS, G. (1996), Les expressions figées en français : noms composés et autres locutions, Paris, Éditions Ophrys.

Gross, G., MASsOussi, T. (2011), «Figement et transparence », in J.-Cl. Anscombre \& S. Mejri (éds), Le figement linguistique : la parole entravée, Paris, Honoré Champion Éditeur, p. 95-108.

Grossmann, F. (2011), « Didactique du lexique : état des lieux et nouvelles orientations », Pratiques 149-150, p. 163-183.

Grossmann, F., TUtin, A. (éds) (2003), Les Collocations : analyse et traitement. Amsterdam : De Werelt. 
KLEIN, J.-R., LAMIROY, B. (2011). « Routines conversationnelles et figement », in J.$\mathrm{Cl}$. Anscombre \& S. Mejri (éds), Le figement linguistique : la parole entravée. $\mathrm{Pa}-$ ris : Honoré Champion, p. 195-213.

MEJRI, S. (2003), «Introduction : polysémie et polylexicalité », Syntaxe et sémantique 5, p. 13-30.

- (2011), «Figement, collocation et combinatoire libre », in J.-Cl. Anscombre \& S. Mejri (éds), Le figement linguistique : la parole entravée, Paris, Honoré Champion Éditeur, p. 63-77.

MEL'ČUK, I. (1993), « La Phraséologie et son rôle dans l'enseignement-apprentissage d'une langue étrangère », Études de linguistique appliquée 92, p. 82-113.

- (2011), "Phrasèmes dans le dictionnaire ", in J.-Cl. Anscombre \& S. Mejri (éds), Le figement linguistique : la parole entravée, Paris, Honoré Champion Éditeur, p. 41-61.

MEL'ČUK, I. A., ClAS, A., POlguÈre, A. (1995), Introduction à la lexicologie explicative et combinatoire, Louvain-la-Neuve, Duculot/ Montréal, AUPELF-UREF.

MONTORO DEL ARCO, E. T. (2011), «Locutions à cases vides, locutions à cases libres, et phénomènes apparentés », in J.-Cl. Anscombre \& S. Mejri (éds), Le figement linguistique : la parole entravée, Paris, Honoré Champion Éditeur, p. 249-265.

SVEnsson, M. H. (2004), Critères de figement : L'identification des expressions figées en français contemporain, Umeå, Umeå Universitet.

TAMBA, I. (2011), «Sens figé : idiomes et proverbes », in J.-Cl. Anscombre \& S. Mejri (éds), Le figement linguistique : la parole entravée, Paris, Honoré Champion Éditeur, p. 109-126.

TUtin, A., GROSSMANN, F. (2002), «Collocations régulières et irrégulières : esquisse de typologie du phénomène collocatif », Revue française de linguistique appliquée, vol. VII, p. 7-25.

VAGUER, C. (2011), «Expressions figées et traduction : langue, culture, traduction automatique, apprentissgae, lexique ", in J.-Cl. Anscombre \& S. Mejri (éds), Le figement linguistique : la parole entravée, Paris, Honoré Champion Éditeur, p. 391412.

VITTOZ, M.-B. (2005), Les locutions en discours : Vers un inventaire de formes idiomatiques dans la langue des affaires Français-Italien, Alessandria, Ed. dell'Orso.

VitToZ-CANUto, M.-B. (1989), De la tête aux pieds : stratégie d'accès au sens de locutions verbales idiomatiques, Tirrenia Stampatori.

\section{Légende des tableaux des Annexes}

Titre $:$ inter. $=$ intermédiaire, ex. $=$ exercices, fr. $=$ français, gram. $=$ grammaire, voc. $=$ vocabulaire, prog. $=$ progressif/ve.

Ed. : C = CLE International, $\mathrm{D}=$ Didier, $\mathrm{H}=$ Hachette Français langue étrangère, $\mathrm{P}=$ Presses universitaires de Grenoble.

Occurr . $=$ occurrences : 0 (aucune) + (sporadiques) ++ (récurrentes) +++ (massives)

Discri. discrimination : 0 (non) $\quad+$ (intermittente $)++$ (régulière $) \quad+++$ (systématique $)$

Décodage : P (paraphrase), S (situation), C (champ sémantique ou champ lexical), CD (chercher dans le dictionnaire), E (explication), AC (approche culturelle), D (défigement)

Encodage : R (reconstitution), Em (emploi), Com (commentaire) 


\section{Annexe 1 : Corpus des manuels analysés}

\begin{tabular}{|c|c|c|c|c|}
\hline $\mathbf{N}^{\circ}$ & Titre & Date & Auteurs & Éd. \\
\hline \multicolumn{5}{|c|}{ Méthodes (12), cahiers d'exercices (7) } \\
\hline 1 & A propos A1 & 2009 & Andant C., Metton C. et al. & $\mathrm{P}$ \\
\hline 2 & A propos, Cahier d'exercices Al & 2009 & Andant C., Metton C. et al. & $\mathrm{P}$ \\
\hline 3 & A propos $A 2$ & 2010 & Carenzi-Vialaneix C. et al. & $\mathrm{P}$ \\
\hline 4 & A propos, Cahier d'exercices $A 2$ & 2010 & Carenzi-Vialaneix C. et al. & $\mathrm{P}$ \\
\hline 5 & A propos $B 1-B 2$ & 2005 & Andant C. et Chalaron M.-L & $\mathrm{P}$ \\
\hline 6 & A propos, Cahier d'exercices B1-B2 & 2005 & Andant C. et Chalaron M.-L & $\mathrm{P}$ \\
\hline 7 & $\begin{array}{l}\text { Communication progressive du français, } \\
\text { débutant }\end{array}$ & 2004 & Miquel C. & $\mathrm{C}$ \\
\hline 8 & Communication progressive du français, inter. & 2004 & Miquel C. & $\mathrm{C}$ \\
\hline 9 & Echo $A 1$ & 2010 & Girardet J., Pécheur J., et al. & $\mathrm{C}$ \\
\hline 10 & Echo Al, Cahier personnel d'apprentissage & 2010 & Girardet J., Pécheur J. & $\mathrm{C}$ \\
\hline 11 & Echo $A 2$ & 2010 & Girardet J., Pécheur J., et al. & $\mathrm{C}$ \\
\hline 12 & Echo A2, Cahier personnel d'apprentissage & 2010 & Girardet J., Gibbe C. & $\mathrm{C}$ \\
\hline 13 & Echo $B 1$ & 2011 & Girardet J., Pécheur J. & $\mathrm{C}$ \\
\hline 14 & Echo B1, Cahier personnel d'apprentissage & 2011 & Girardet J., Pécheur J. & $\mathrm{C}$ \\
\hline 15 & Echo B2 & 2010 & Girardet J., Gibbe C. & $\mathrm{C}$ \\
\hline 16 & Echo B2, Cahier personnel d'apprentissage & 2010 & Girardet J., Callet S. & $\mathrm{C}$ \\
\hline 17 & Le nouvel édito B2 & 2010 & Brillant C., Bazou V., et al. & $\mathrm{D}$ \\
\hline 18 & Scénario 1 & 2008 & Dubois A.-L., et al. & $\mathrm{H}$ \\
\hline 19 & Scénario 2 & 2008 & Guilloux M., Daill E. & $\mathrm{H}$ \\
\hline \multicolumn{5}{|c|}{ Français sur objectifs spécifiques (9), cahiers d'exercices (2) } \\
\hline 20 & Objectif express 1 & 2006 & Dubois A.-L., Tauzin B. & $\mathrm{H}$ \\
\hline 21 & Objectif express 1, Cahier d'activités & 2006 & Dubois A.-L., Tauzin B. & $\mathrm{H}$ \\
\hline 22 & Objectif express 2 & 2009 & Dubois A.-L., Tauzin B. & $\mathrm{H}$ \\
\hline 23 & Objectif express 2, Cahier d'activités & 2009 & Gillet N., Tauzin B. & $\mathrm{H}$ \\
\hline 24 & Le français du monde du travail & 2004 & Cloose E. & $\mathrm{P}$ \\
\hline 25 & Le français pour les sciences & 2004 & Tolas J. & $\mathrm{P}$ \\
\hline 26 & Science-technique.com & 2005 & Lahmidi Z. & $\mathrm{C}$ \\
\hline 27 & Travailler en français en entreprise 1 & 2010 & Gillman B. & $\mathrm{D}$ \\
\hline 28 & Travailler en français en entreprise 2 & 2009 & Cherifi S., et al & $\mathrm{D}$ \\
\hline \multicolumn{5}{|c|}{ Vocabulaire (7) } \\
\hline 29 & Le chemin des mots & 2005 & Dumarest D., Morsel M.-H. & $\mathrm{P}$ \\
\hline 30 & Dites-moi un peu & 2005 & Hingue A.-M., Ulm K. & $\mathrm{P}$ \\
\hline 31 & Le français par les textes I & 2003 & Barthe M., et al. & $\mathrm{P}$ \\
\hline 32 & Le français par les textes II & 2003 & Barthe M., Chovelon B. & $\mathrm{P}$ \\
\hline 33 & Vocabulaire progressif du français, débutant & 2001 & Miquel C. & $\mathrm{C}$ \\
\hline 34 & Vocabulaire progressif du français, inter. & 1997 & Miquel C., Goliot-Lété A. & $\mathrm{C}$ \\
\hline 35 & Vocabulaire progressif du français, avancé & 1999 & Miquel C. & $\mathrm{C}$ \\
\hline \multicolumn{5}{|c|}{ Grammaire (7) } \\
\hline 36 & Grammaire du français A1-A2 & 2005 & Bérard E. & $\mathrm{D}$ \\
\hline 37 & Grammaire pratique du français & 2000 & Delatour Y., et al. & $\mathrm{H}$ \\
\hline 38 & Grammaire des premiers temps, vol. 1 & 2004 & Abry D., Chalaron M.-L. & $\mathrm{P}$ \\
\hline 39 & Grammaire des premiers temps, vol. 2 & 2003 & Abry D., Chalaron M.-L. & $\mathrm{P}$ \\
\hline 40 & $\begin{array}{l}\text { Grammaire progressive du français, } \\
\text { débutant }\end{array}$ & 1997 & Grégoire M. & $\mathrm{C}$ \\
\hline 41 & Grammaire progressive du français, inter. & 1995 & Grégoire M., Thiévenaz O. & $\mathrm{C}$ \\
\hline 42 & Grammaire progressive du français, avancé & 1997 & Boularès M., Frérot J.-L. & $\mathrm{C}$ \\
\hline
\end{tabular}


Annexe 2 : Occurrences et traitement des collocations

\begin{tabular}{|c|c|c|c|c|c|}
\hline $\mathbf{N}^{\circ}$ & Titre & Occurr. & Discri. & Décodage & Encodage \\
\hline 1 & A propos A1 & ++ & + & $S$ & $E m$ \\
\hline 2 & A propos, Cahier d'ex. Al & + & + & & \\
\hline 3 & A propos A2 & ++ & + & $S$ & $E m$ \\
\hline 4 & A propos, Cahier d'ex. A2 & + & + & & \\
\hline 5 & A propos $B 1-B 2$ & ++ & + & $S$ & Em \\
\hline 6 & A propos, Cahier d'ex. B1-B2 & ++ & ++ & $S-C$ & $E m$ \\
\hline 7 & Communication prog. 1 & + & ++ & $S$ & $R-E m$ \\
\hline 8 & Communication prog. 2 & + & ++ & $S$ & $R-E m$ \\
\hline 9 & Echo A1 & ++ & + & $S$ & Em \\
\hline 10 & Echo A1, Cahier personnel & $0 /+$ & 0 & & \\
\hline 11 & Echo $A 2$ & + & + & $S$ & $R-E m$ \\
\hline 12 & Echo A2, Cahier personnel & ++ & + & $S$ & \\
\hline 13 & Echo B1 & ++ & + & $S$ & Em \\
\hline 14 & Echo B1, Cahier personnel & ++ & + & $S$ & $R-E m$ \\
\hline 15 & Echo B2 & ++ & + & $S$ & $R-E m$ \\
\hline 16 & Echo B2, Cahier personnel & ++ & + & & \\
\hline 17 & Le nouvel édito $B 2$ & + & + & $S-P$ & $E m$ \\
\hline 18 & Scénario 1 & ++ & + & $S-C$ & $R-E m$ \\
\hline 19 & Scénario 2 & ++ & + & $S-C$ & Em \\
\hline 20 & Objectif express 1 & ++ & ++ & $S-E x-C$ & $E m$ \\
\hline 21 & Objectif express 1, Cahier d'ex. & + & ++ & & $R$ \\
\hline 22 & Objectif express 2 & ++ & + & $S-E x-C$ & $E m$ \\
\hline 23 & Objectif express 2, Cahier d'ex. & ++ & ++ & $S$ & $R$ \\
\hline 24 & Le fr. du monde du travail & ++ & ++ & $E x-C$ & $E m$ \\
\hline 25 & Le fr. pour les sciences & ++ & ++ & $E x-C$ & $E m$ \\
\hline 26 & Science-technique.com & + & + & $P-E x-C$ & $R-E m$ \\
\hline 27 & Travailler en français 1 & ++ & ++ & $P-S-C$ & $R-E m$ \\
\hline 28 & Travailler en français 2 & ++ & ++ & $P-S-C$ & $E m$ \\
\hline 29 & Le chemin des mots & +++ & +++ & $P-E x-C-C D-D$ & $R-E m$ \\
\hline 30 & Dites-moi un peu & + & + & C & Em \\
\hline 31 & Le fr. par les textes I & + & ++ & $S-E x-C$ & $E m$ \\
\hline 32 & Le fr. par les textes II & ++ & + & $S-E x-C$ & $E m$ \\
\hline 33 & Vocabulaire prog. 1 & ++ & +++ & $S-E x-C$ & $R-E m$ \\
\hline 34 & Vocabulaire prog. 2 & ++ & +++ & $S-E x-C$ & $R-E m$ \\
\hline 35 & Vocabulaire prog. 3 & ++ & +++ & $S-E x-C$ & $R-E m$ \\
\hline 36 & Gram. du français A1-A2 & + & + & $S$ & $E m$ \\
\hline 37 & Gram. pratique du fr. & $0 /+$ & 0 & & \\
\hline 38 & Gram. des premiers temps 1 & ++ & + & $S$ & $E m$ \\
\hline 39 & Gram. des premiers temps 2 & ++ & + & $S$ & $R-E m$ \\
\hline 40 & Gram. prog. du fr. 1 & $0 /+$ & 0 & & \\
\hline 41 & Gram. prog. du fr. 2 & + & + & & $E m$ \\
\hline 42 & Gram. prog. du fr. 3 & ++ & ++ & & $R-E m$ \\
\hline
\end{tabular}


Annexe 3 : Occurrences et traitement des expressions imagées et proverbes

\begin{tabular}{|c|c|c|c|c|c|}
\hline $\mathbf{N}^{\circ}$ & Titre & Occurr. & Discri. & Décodage & Encodage \\
\hline 1 & A propos A1 & 0 & & & \\
\hline 2 & A propos, Cahier d'ex. Al & 0 & & & \\
\hline 3 & A propos A2 & $0 /+$ & + & & \\
\hline 4 & A propos, Cahier d'ex. A2 & + & +++ & $P$ & $R$ \\
\hline 5 & A propos $B 1-B 2$ & + & +++ & & \\
\hline 6 & A propos, Cahier d'ex. B1-B2 & + & +++ & $P-E x$ & Com, Em \\
\hline 7 & Communication prog. 1 & + & ++ & $S-E x$ & \\
\hline 8 & Communication prog. 2 & + & ++ & $S-E x$ & \\
\hline 9 & Echo A1 & $0 /+$ & + & $P$ & \\
\hline 10 & Echo A1, Cahier personnel & 0 & & & \\
\hline 11 & Echo A2 & + & + & $P-S-C D-E x$ & \\
\hline 12 & Echo A2, Cahier personnel & + & + & $P-S-C D-E x$ & \\
\hline 13 & Echo B1 & + & + & $P-S-C D-E x-D$ & $E m$ \\
\hline 14 & Echo B1, Cahier personnel & + & + & $P-E x$ & Em \\
\hline 15 & Echo B2 & ++ & ++ & $P-S-C D-E x$ & $R-E m$ \\
\hline 16 & Echo B2, Cahier personnel & ++ & + & $P-S-C D-E x$ & $R$ \\
\hline 17 & Le nouvel édito B2 & ++ & ++ & $P-E x-D$ & $E m$ \\
\hline 18 & Scénario 1 & + & + & $P-A C-D$ & \\
\hline 19 & Scénario 2 & + & + & $P-C D-A C$ & \\
\hline 20 & Objectif express 1 & 0 & & & \\
\hline 21 & Objectif express 1, Cahier d'ex. & + & +++ & $P-D$ & \\
\hline 22 & Objectif express 2 & $0 /+$ & + & Ex & \\
\hline 23 & Objectif express 2, Cahier d'ex. & 0 & & & \\
\hline 24 & Le fr. du monde du travail & + & 0 & & \\
\hline 25 & Le fr. pour les sciences & 0 & & & \\
\hline 26 & Science-technique.com & + & 0 & & \\
\hline 27 & Travailler en fr. 1 & + & 0 & & \\
\hline 28 & Travailler en fr. 2 & + & 0 & & \\
\hline 29 & Le chemin des mots & +++ & +++ & $P-S-A C-C D-D$ & Em \\
\hline 30 & Dites-moi un peu & +++ & +++ & & Em-Com \\
\hline 31 & Le fr.par les textes I & ++ & ++ & $P-S-E x$ & \\
\hline 32 & Le fr.par les textes II & + & + & $P-S-E x$ & \\
\hline 33 & Voc. prog. 1 & $0 /+$ & +++ & $P-S-E x$ & \\
\hline 34 & Voc. prog. 2 & + & +++ & $P$ & $E m$ \\
\hline 35 & Voc. prog. 3 & ++ & +++ & $P-S$ & $R-E m$ \\
\hline 36 & Gram. du fr. A1-A2 & + & + & $C D$ & \\
\hline 37 & Gram. pratique du fr. & + & + & $D$ & \\
\hline 38 & Gram. des premiers temps 1 & + & + & $C D-A C$ & $R$ \\
\hline 39 & Gram. des premiers temps 2 & + & + & & \\
\hline 40 & Gram.prog. du fr. 1 & 0 & & & \\
\hline 41 & Gram. prog. du fr. 2 & 0 & & & \\
\hline 42 & Gram. prog. du fr. 3 & + & 0 & & \\
\hline
\end{tabular}

